\title{
Special Issue: Nine Basic Questions on the Origins of Life, International School of Complexity, Erice (Italy), 1 to 6 October 2006.
}

\author{
Alan W. Schwartz \\ Published online: 9 August 2007 \\ (C) Springer Science + Business Media B.V. 2007
}

This issue of Origins of Life and Evolution of Biospheres contains a collection of short papers which summarize the presentations from the "International School of Complexity", held in Erice and organized by Pasquale Stano and Pier Luigi Luisi. The organizers not only assembled a superlative group of discussants for the workshop, but have admirably edited the results which summarize the contributions and conclusions. I wish to thank both of them most sincerely for preparing the event and especially for the hard work of editing the resulting manuscripts and making this special issue possible.

\footnotetext{
A. W. Schwartz $(\bowtie)$

Radboud University, Nijmegen, The Netherlands

e-mail: alan@sci.ru.nl
} 